Mathematical Research Letters 6, 99-105 (1999)

\title{
ON REPRESENTATIONS OF COMPLEX HYPERBOLIC LATTICES
}

\author{
Mutao Wang
}

\begin{abstract}
The following superrigidity type theorem for complex hyperbolic lattices is proved in this paper. Let $X=\Gamma \backslash B^{n}$ be a compact complex ball quotient, $n=2$ or 3 . Suppose $H^{1,1}(X, C) \cap H^{2}(X, Z)$ is generated by the Kähler class of $X$. Then any representation of $\Gamma$ in $G L(n+1, C)$ can either be deformed to a unitary representation or be extended to a homomorphism from $S U(n, 1)$ into $G L(n+1, C)$.
\end{abstract}

\section{Introduction}

Let $G$ be a connected non-compact semi-simple Lie group. Recall that a discrete subgroup $\Gamma \subset G$ is called a lattice in $G$ if $\Gamma \backslash G$ is of finite volume. A lattice should in spirit inherit a lot of characteristics of the embient group. If $G$, in addition, admits some algebraic group structure, a family of discrete subgroups called arithmetic subgroups can be constructed. It was proved by Borel and Harish-Chandra [2] that all arithmetic subgroups are lattices. Nevertheless, it is far from obvious that the converse could be true, though this statement for most semisimple Lie groups was conjectured by Selberg and Piatetski-Shapiro in the sixties.

Margulis' celebrated arithmeticity theorem gives an affirmative answer to this conjecture and asserts that irreducible lattices in any semisimple Lie group of real rank greater than or equal to two are arithmetic subgroups. His theorem left unanswered the arithmeticity of lattices in real rank one groups. There are essentially four real rank one simple Lie groups: $S O(n, 1), S U(n, 1), S p(n, 1)$

and $F_{4}^{(-20)}$. The arithmeticity of lattices in the latter two groups were finally proved in the ninties due to the work of Corlette [4] and Gromov-Schoen [9]. While for the former two groups, it was long known that non-arithmetic lattices do exist. In $S O(n, 1)$, such examples were constructed by Makarov [13], Vinberg [25], and Gromov-Piatetski-Shapiro [8] for all $n$.

In this article, we study lattices in $S U(n, 1)$. The first non-arithmetic lattice in $S U(2,1)$ was constructed by Mostow [16], [17] as a discrete group generated by reflections along the sides of a polyhedra in the complex two ball. In [6], a familly of complex hyperbolic lattices were constructed by Deligne and Mostow as the fundamental groups of certain compactifications of moduli spaces of marked points on the Riemann sphere. Later, Thurston [23] studied the moduli spaces

Received May 27, 1998. Revised August 14, 1998. 
of Euclidean cone metrics on the sphere and more non-arithmetic examples were obtained in this way.

Among their lists there are non-arithmetic lattices in $S U(2,1)$ and $S U(3,1)$. It is therefore important to determine arithmetic lattices in these groups. In [20], the author addressed a conjecture due to Rogawski, where a criterion of arithmeticity of cocompact latttices in $S U(2,1)$ was given in terms of the topology of the quotient manifold. Namely,

Conjecture 1.1. Let $X=\Gamma \backslash B^{2}, \Gamma \subset S U(2,1)$ be a compact ball quotient. If $b_{1}(X)=0$ and $H^{1,1}(X) \cap H^{2}(X, Q)=Q$, then $\Gamma$ is arithmetic.

Such examples were first given by Mumford [18] by the method of $p$-adic uniformization. In particular, he constructed an arithmetic complex hyperbolic surfaces with the same betti numbers as $C P^{2}$.

According to Margulis' theorem, the arithmeticity of a lattice can be established through the Archimedean and non-Archimedean superrigidity of the lattice. In this paper, the following Archimedean superrigidity type theorem was proved.

Theorem 1.2. Let $X=\Gamma \backslash B^{n}(n=2,3)$ be a smooth compact complex ball quotient. Suppose $H^{1,1}(X, C) \cap H^{2}(X, Z)$ is generated by the Kähler class of $X$. Then any representation of $\Gamma$ in $G L(n+1, C)$ can be either deformed to a unitary representation or extended to a homomorphism from $S U(n, 1)$ into $G L(n, C)$.

The case of $\Gamma \backslash B^{2}$ was proved in [20] under an extra assumption that $b_{1}(X)=$ 0 . The argument there depends on complex variations of Hodge structures and the stability of Higgs bundles. The key point is the restriction of Higgs bundles to complex curves and it seems only applicable to the complex hyperbolic surface case. Our proof also makes use of the idea of complex variations of Hodge structures. The key point is replaced by a harmonic map argument. Harmonic maps were used by Corlette and Gromov-Schoen in their work leading to arithmeticity of lattices in $S p(n, 1)$ and $F_{4}^{(-20)}$. This approach was also used to give a new proof of the Margulis superrigidity in the cocompact case by Jost-Yau [10] and independently, Mok-Siu-Yeung [15].

The present article is organized as the following. In $\S 2$ we discuss harmonic maps and Simpson's theory of complex variations of Hodge structures. In $\S 3$, we prove the main theorem.

I would like to thank Professor S.-T. Yau for suggesting this problem and constant encouragement. I also benefitted from discussion with Z. Lu, C-L Wang, and K. Zuo. 


\section{Harmonic maps and complex variation of Hodge structures}

In this section, $G$ denotes a non-compact semi-simple Lie group without compact factor. We assume $G$ is isomorphic to the real points of a semisimple algebraic group over $R$. $K$ denotes a maximal compact subgroup of $G$, so $G / K$ is a Riemannian symmetric space. Let $X$ be a compact Riemannian manifold with fundamental group $\pi_{1}(X)$ and $\tilde{X}$ the universal covering of $X$. Let $\rho: \pi_{1}(X) \rightarrow G$ be a group homomorphism. The following theorem is well known.

Theorem 2.1. (Corlette [5], Labourie [12], Jost-Yau [11]) If the Zariski closure of the image of $\rho$ is a reductive subgroup in $G$, then there exists a harmonic map $f: \tilde{X} \rightarrow G / K$ which is equivariant with respect to $\rho$, i.e. $f(\gamma x)=\rho(\gamma) f(x)$ for all $x \in \tilde{X}$ and $\gamma \in \pi_{1}(X)$.

If $X$ is further assumed to be Kähler, then we can actually say more about the harmonic map. First let's recall Simpson's theory of complex variations of Hodge structures from [22].

Definition 2.2. A complex variation of Hodge structures is a smooth complex vector bundle $V$ with a decomposition $V=\oplus_{r+s=w} V^{r, s}$, a flat connection $D$ satisfying Griffiths' transversality condition

$$
\begin{aligned}
& D=\partial+\bar{\partial}+\theta+\bar{\theta}: V^{r, s} \rightarrow \\
& A^{1,0}\left(V^{r, s}\right) \oplus A^{0,1}\left(V^{r, s}\right) \oplus A^{1,0}\left(V^{r-1, s+1}\right) \oplus A^{0,1}\left(V^{r+1, s-1}\right)
\end{aligned}
$$

and a parallel Hermitian form which makes the Hodge decomposition orthogonal and which on $V^{r, s}$ is positive definite if $r$ is even and negative definite if $r$ is odd.

If we fix a base point on $X$, then the monodromy representation of the flat connection $D$ gives a representation of $\pi_{1}(X)$ into the group $U(p, q)$ with $p+q=$ the rank of $V$.

The theorem of Simpson asserts that such representations appear in every component of the space of representions of $\pi_{1}(X)$ in $G L(n, C)$.

Theorem 2.4. Let $X$ be a compact Kähler manfold, then any representaion $\rho: \pi_{1}(X) \rightarrow G L(n, C)$ can be deformed to a representation which comes from a complex variation of Hodge structures.

By Corollary 4.2 in [22], the monodromy representation of the flat connection $D$ associated to a complex variation of Hodge structures is reductive. Therefore it also gives an equivariant harmonic map $f$ from $\tilde{X}$ into $U(p, q) / U(p) \times U(q)$. On the other hand, by Griffith's theory [7], such a variation of Hodge structure gives a horizontal holomorphic map $\tilde{f}$ from $\tilde{X}$ into the associated period domain $D$. $D$ is a homogeneous Kähler manifold of the form $U(p, q) / H$, where $H \subset U(p, q)$ is a compact subgroup. There is a natural Riemannian submersion $\pi: D \rightarrow$ $U(p, q) / U(p) \times U(q)$. According to a theorem of Borel [1], this $\pi$ is holomorphic since $U(p, q) / U(p) \times U(q)$ is Hermitian symmetric. As holomorphic maps on 
Kähler manifolds are harmonic, by the uniqueness of harmonic maps, $\pi \circ \tilde{f}=f$. We have prove the following proposition.

Proposition 2.5. Let $X$ be a compact Kähler manifold, then any representation $\rho: \pi_{1}(X) \rightarrow G L(n, C)$ can be deformed to a new representation $\rho^{\prime}$ whose associated equivariant harmonic map from $\tilde{X}$ into $U(p, q) / U(p) \times U(q)$ is holomorphic.

\section{Proof of the main theorem}

Now we proceed to prove Theorem 1.2.

Proof. Let $X=\Gamma \backslash B^{3}$ be a compact complex ball quotient. $X$ endowed with the canonical Kähler form $\omega_{X}$ is a compact Kähler manifold. Suppose $H^{1,1}(X, C)$ $\cap H^{2}(X, Z)$ is generated $\omega_{X}$. Applying Simpson's theorem to our case, the representation $\rho$ of $\Gamma$ into $G L(4, C)$ is deformable to a new representation $\rho^{\prime}$ into one of $U(4), U(3,1)$, or $U(2,2)$.

In the $U(4)$ case, we are done. The infinitesimal deformation space of $\Gamma$ in $G L(4, C)$ of the new representation $\rho^{\prime}$ is parametrized by the group cohomology $H^{1}(\Gamma, \mathfrak{g l}(4, C))$, where the action of $\Gamma$ on $\mathfrak{g l}(4, C)$ factors through the homomorphism of $\Gamma$ into $U(4)$ and the adjoint action of $U(4)$ on $\mathfrak{g l}(4, C)$ and is therefore a unitary representation. If we were able to show this cohomology group is trivial, it would implied the deformed representation is conjugate to the original representation. This would show the original representation is also unitary. However we are not able to achieve this at this moment. See [3] for more details on this group cohomology.

In the $U(3,1)$ case, we prove that the harmonic map is actually totally geodesic.

Let $\omega_{M}$ denotes the canonical Kähler form on $M=U(3,1) / U(3) \times U(1)$. The holomorphic map $f$ maps $\tilde{X}$ to $M$ equivariantly. Therefore $f^{*} \omega_{M}$ descends to $X$. By assumption,

$$
f^{*} \omega_{M}=k \omega_{X}+C,
$$

where $k$ is a non-negative integer and $C$ is an exact form. We claim that we may assume $k$ is non-zero. If $k$ were zero then $C$ would be a non-negative hermitian $(1,1)$ form representing a trivial cohomology class.

By the definiteness of $C, C \wedge \omega_{X}^{n-1}=\beta \omega_{X}^{n}$ for some non-negative function $\beta$ which equals pointwise to the sum of eigenvalues of $C$. But $\int C \wedge \omega_{X}^{n-1}=0$, this implies $\beta$ is identically zero. $C$ is indeed a zero form and $f^{*} \omega_{M}$ is identically zero which implies $f$ is a constant map. Thus the image of $\rho$ lies in some compact subgroup of $U(3,1)$. From now on we assume $k \geq 1$.

On the other hand, by Schwarz lemma, we have

$$
\omega_{X} \geq f^{*} \omega_{M} .
$$

Combining this with (3.1) and notice that $k \geq 1$, it follows that $-C$ is again a non-negative $(1,1)$ exact form. By the same reason, we get $C=0$ and $k=1$, 
or $\omega_{X}=f^{*} \omega_{M}$. It follows that $f$ is an isometry. We apply the Bochner formula for harmonic maps in [10] and conclude $\nabla d f=0$. This is equivalent to saying $f$ is totally geodesic.

Now a totally geodesic map can be lifted to a continuous homomorphism from $S U(3,1)$ to $U(3,1)$. This homomorphism is conjugate to the standard inclusion and the new representation $\rho^{\prime}$ is conjugate to the standard inclusion of $\Gamma$ into $U(3,1)$. The infinitesimal deformation space of $\rho^{\prime}$ in $G L(4, C)$ is parametrized by the group cohomoloy $H^{1}(\Gamma, \mathfrak{g l}(4, C))$, where the action of $\Gamma$ on $\mathfrak{g l}(4, C)$ factors through the standard incl sion of $\Gamma$ into $U(3,1)$ and the adjoint action of $U(3,1)$ on $\mathfrak{g l}(4, C)$. This group cohomology is trivial by a theorem of Raghunathan [19], see also Proposition 6.5 in [3]. This implies $\rho$ is conjugate to $\rho^{\prime}$.

In the $U(2,2)$ case, we prove that the harmonic map does not exist. This is done by a total Chern class calculation. First of all, $f$ must be a holomorphic immersion regarding the fact that a contracting complex curve or complex surface in $X$ represents a nontrivial homology class whose Poincare dual is different from any multiple of the class of $\omega_{X}^{2}$ or $\omega_{X}$.

Now we calculate the total Chern class of $U(p, q) / U(p) \times U(q)$ using the proportionality principle. The compact dual of $U(p, q) / U(p) \times U(q)$ is the complex Grassmannian $G_{p, q}$. The total Chern class of the complex Grassmannian can be computed as the following. We assume $p \leq q$. Let $S$ denote the tautological bundle over it and $Q$ the quotient bundle of the trivial bundle of rank $p+q$ by $S$.

$$
0 \rightarrow S \rightarrow C^{p+q} \rightarrow Q \rightarrow 0
$$

The tangent bundle of $G_{p, q}$ is isomorphic to the bundle $\operatorname{Hom}(S, Q)=S^{*} \otimes Q$. Therefore,

$$
c\left(S^{*} \otimes Q\right)=c\left(S^{*} \otimes C^{p+q}\right) / c\left(S^{*} \otimes S\right)=c\left(S^{*}\right)^{p+q} / c\left(S^{*} \otimes S\right) .
$$

$c\left(S^{*} \otimes S\right)$ can be computed using the formula of the Chern character and the fact that $\operatorname{ch}\left(S^{*} \otimes S\right)=\operatorname{ch}\left(S^{*}\right) \operatorname{ch}(S)$. Using the propotionality principle, it is not hard to write the total Chern class of the tangent bundle of $U(p, q) / U(p) \times U(q)=M$ in terms of the Chern classes of $S$.

Now we specialize to the case when $p=q=2$. We consider the pullback of the total Chern class of $M$ which again descends to $X$. Since the cohomology ring of $X$ is generated by $\omega_{X}$. The pullback of the total Chern class of $M$ involves two integral variables $k_{1}$, and $k_{2}$, where $f^{*} c_{1}(S)=k_{1} \omega_{X}$ and $f^{*} c_{2}(S)=k_{2} \omega_{X}^{2}$. The total Chern class of the normal bundle, as a line bundle, only involves a single integral variable $k_{3}$ with $c_{1}(N)=k_{3} \omega_{X}$. The equality

$$
c(T X) c(N)=c\left(f^{*} T M\right)
$$

of total Chern classes gives an equation satisfied by the three integral variables $k_{1}, k_{2}$ and $k_{3}$. It is a straightforward calculation to check this equation does not allow any integral solutions. 
The case when $X=\Gamma \backslash B^{2}$ can be proved in a similar way and we omit it here.

\section{References}

[1] A. Borel, Kählerian coset spaces of semi-simple Lie groups, Proc. Nat. Acad. Sci. U.S.A. 40 (1954), 1147-1151.

[2] A. Borel and Harish-Chandra, Arithmetic subgroups of algebraic groups, Ann. of Math. (2) 75 (1962), 485-535.

[3] A. Borel, and N. Wallach, Continuous cohomology, discrete subgroups, and representations of reductive groups, Ann. of Math. Studies, 94., Princeton University Press, Princeton, N.J., 1980.

[4] K. Corlette, Archimedean superrigidity and hyperbolic geometry, Ann. of Math. (2) 135 (1992), 165-182.

[5] — Flat G-bundle with canonical metrics, J. Differential Geom. 28 (1988), 361-382.

[6] P. Deligne and G.D. Mostow, Monodromy of hypergeometric functions and non-lattice integral monodromy, Inst. Hautes Études Sci. Publ. Math. 63 (1986), 5-89.

[7] P. Griffiths, Periods of integrals on algebraic manifolds I. Construction and properties of the modular varieties, Amer. J. Math. 90 (1968), 568-626,

Periods of integrals on algebraic manifolds II. Local study of the period mapping, Amer. J. Math. 90 (1968), 805-865,

Periods of integrals on algebraic manifolds III. Some global differential-geometric properties of the period mapping, Inst. Hautes Études Sci. Publ. Math. 38 (1970), 125-180.

[8] M. Gromov and I. Piatetski-Shapiro, Non-arithmetic groups in Lobachevsky spaces, Inst. Hautes Études Sci. Publ. Math. 65 (1988), 93-103.

[9] M. Gromov and R. Schoen, Harmonic maps into singular spaces and p-adic superrigidity for lattices in groups of rank one, Inst. Hautes Études Sci. Publ. Math. 76 (1992), 165-246.

[10] J. Jost and S.-T. Yau, Harmonic maps and superrigidity, in Differential geometry: partial differential equations on manifolds (Los Angeles, CA, 1990), Proc. Sympos. Pure Math., 54, Part 1, Amer. Math. Soc., Providence, RI, 1993, pp. 245-280.

[11] J. Jost and S.-T. Yau, Harmonic maps and group representations, in Differential Geometry (B. Lawson and K. Tennenblat, eds.), Pitman Monographs Surveys Pure Appl. Math., 52, Longman Sci. Tech., Harlow, 1991, pp. 241-260.

[12] F. Labourie, Existence d'applications harmoniques tordues à valeurs dans les variétés à courboure négative, Proc. Amer. Math. Soc. 111 (1991), 877-882.

[13] V.S. Makarov, On a certain class of discrete groups of Lobachevsky space having an infinite fundamental region of finite measure, Soviet Math. Dokl. 7 (1966), 328-331.

[14] G.A. Margulis, Discrete subgroups of semisimple Lie groups, Ergebnisse der Mathematik und ihrer Grenzgebiete (3), 17., Springer-Verlag, Berlin, 1991.

[15] N. Mok, Y.-T. Siu, and S.K. Yeung, Geometric superrigidity, Invent. Math., 113 (1) (1993). 57-83.

[16] G.D. Mostow, Existence of a non-arithmetic lattice in SU(2,1), Proc. Nat. Acad. Sci. USA 78 (1981), 5948-5950.

[17] _ On a remarkable class of polyhedra in complex hyperbolic space, Pacific J. Math. 86 (1980), 171-276.

[18] D. Mumford, An algebraic surface with $K$ ample, $K^{2}=9, p_{g}=q=0$, Compositio Math.

[19] M.S. Raghunathan, On the first cohomology of discrete subgroups of semi-simple Lie groups, Amer. J. Math. 87 (1965), 103-139.

[20] A. Reznikov, Simpson's theory and superrigidity of complex hyperbolic lattices, C. R. Acad. Sci. Paris Sér. I Math., 320 (1995), 1061-1064.

[21] J. Rogawski, Automorphic representations of unitary groups in three variables, Annals of Mathematics Studies, 123., Princeton University Press, Princeton, N.J., 1990. 
[22] C. Simpson, Higgs bundles and local systems, Inst. Hautes Études Sci. Publ. Math., 75 (1992), 5-95.

[23] W. Thurston, Shapes of polyhedra, preprint.

[24] D. Toledo, Rigidity theorems in Kähler Geometry and Fundamental Groups of Varieties, preprint, 1996.

[25] E.B. Vinberg, Discrete groups generated by reflections in Lobachevsky spaces, Math. USSR Sb. 1 (1967), 429-444.

[26] R. Zimmer, Ergodic theory and semisimple groups, Monographs in Mathematics, 81., Birkhaüser Verlag, Basel-Boston, Mass., 1984.

Department of Mathematics, Harvard University, Cambridge, MA 02138

E-mail address: mtwangmath.harvard.edu 